University of Wollongong

Research Online

Faculty of Engineering and Information

Faculty of Engineering and Information

Sciences - Papers: Part A

Sciences

$1-1-2011$

Octave spanning supercontinuum in an As2S3 taper using ultralow pump pulse energy

Darren D. Hudson

University of Sydeny

Stephen A. Dekker

University of Sydeny

Eric C. Magi

University of Sydney

Alexander C. Judge

University of Sydeny

Stuart D. Jackson

University of Sydney

See next page for additional authors

Follow this and additional works at: https://ro.uow.edu.au/eispapers

Part of the Engineering Commons, and the Science and Technology Studies Commons

Research Online is the open access institutional repository for the University of Wollongong. For further information contact the UOW Library: research-pubs@uow.edu.au 


\title{
Octave spanning supercontinuum in an As2S3 taper using ultralow pump pulse energy
}

\begin{abstract}
An octave spanning spectrum is generated in an As2S3 taper via $77 \mathrm{pJ}$ pulses from an ultrafast fiber laser. Using a previously developed tapering method, we construct a $1.3 \mu \mathrm{m}$ taper that has a zerodispersion wavelength around $1.4 \mu \mathrm{m}$. The low two-photon absorption of sulfide-based chalcogenide fiber allows for higher input powers than previous efforts in selenium-based chalcogenide tapered fibers. This higher power handling capability combined with input pulse chirp compensation allows an octave spanning spectrum to be generated directly from the taper using the unamplified laser output.
\end{abstract}

\section{Keywords}

pulse, pump, taper, energy, octave, ultralow, spanning, supercontinuum, as 2 s 3

Disciplines

Engineering | Science and Technology Studies

\section{Publication Details}

Hudson, D., Dekker, S., Magi, E., Judge, A., Jackson, S., Li, E., Sanghera, J., Shaw, L., Aggarwal, I. \& Eggleton, B. (2011). Octave spanning supercontinuum in an As2S3 taper using ultralow pump pulse energy. Optics Letters, 36 (7), 1122-1124.

\section{Authors}

Darren D. Hudson, Stephen A. Dekker, Eric C. Magi, Alexander C. Judge, Stuart D. Jackson, Enbang Li, J S. Sanghera, L B. Shaw, I D. Aggarwal, and Benjamin J. Eggleton 


\title{
Octave spanning supercontinuum in an $\mathrm{As}_{2} \mathrm{~S}_{3}$ taper using ultralow pump pulse energy
}

\author{
Darren D. Hudson, ${ }^{1, *}$ Stephen A. Dekker, ${ }^{1}$ Eric C. Mägi, ${ }^{1}$ Alexander C. Judge, ${ }^{1}$ Stuart D. Jackson, ${ }^{1}$
Enbang Li, ${ }^{1}$ J. S. Sanghera, ${ }^{2}$ L. B. Shaw ${ }^{2}$ I. Sa \\ Enbang Li, ${ }^{1}$ J. S. Sanghera, ${ }^{2}$ L. B. Shaw, ${ }^{2}$ I. D. Aggarwal, ${ }^{2}$ and Benjamin J. Eggleton ${ }^{1}$ \\ ${ }^{1}$ Centre for Ultrahigh-Bandwidth Devices for Optical Systems, Institute for Photonics and Optical Science,
School of Physics, The University of Sydney, NSW \\ ${ }^{2}$ Naval Research Laboratory, Code 5620, Washington 2006, Australia \\ ${ }^{\star}$ Corresponding author: hudson@physics.usyd.C. 20375, USA
}

Received January 28, 2011; revised February 21, 20 posted March 1, 2011 (Doc. ID 141697); published accepted February 22, 2011;

An octave spanning spectrum is generated in an $\mathrm{As}_{2} \mathrm{~S}_{3}$ taper via $77 \mathrm{pJ}$ (arch 22, 2011

previously developed tapering method, we construct a $1.3 \mu \mathrm{m}$ taper that has a zero-dispersion wiber laser. Using a
$1.4 \mu \mathrm{m}$. The low two-photon absorption of sulfide-based $1.4 \mu \mathrm{m}$. The low two-photon absorption of sulfide-based chalcogenide fiber allow-dispersion wavelength around previous efforts in selenium-based chalcogenide tapered fibers. This higher power for higher input powers than with input pulse chirp compensation allows an octave spanning spectrum to be

using the unamplified laser output. (C) 2011 Optical Society of America
OCIS codes: $320.6629,190.4370$.

The generation of supercontinuum spectra in optical fiber has played an important role in many technologies, such as optical coherence tomography [1], spectroscopy [2], and frequency metrology [3]. In particular, when an octave of bandwidth is achieved, a mode-locked laser can be used as a frequency comb generator via the $f-2 f$ interferometer technique [4]. However, achieving such large bandwidths requires pulse energies on the order of nanojoules, thus imposing constraints on the requisite power level. The typical arrangement for generating an octave with an erbium-based mode-locked laser involves dispersion compensation and an optical amplifier before entering a section of highly nonlinear fiber. On top of the added complexity, the amplifier adds amplified spontaneous emission noise to the output, thereby reducing the ultimate signal-to-noise achievable in an optical frequency measurement [5].

In this Letter, we present a sulfide-based chalcogenide taper that is capable of generating over one octave of bandwidth at a pulse energy of only $77 \mathrm{pJ}$, using a standard, unamplified mode-locked erbium fiber laser. Furthermore, at higher pulse energies, the octave spanning spectrum becomes extremely flat, with an octave contained within the $-10 \mathrm{~dB}$ points.

Apart from the potential usefulness of chalcogenidebased glasses in the mid-IR wavelength range [6] (where they are transparent), the chalcogenides also have a stronger nonlinearity than standard silica-based fibers in the telecommunications wavelength range. This high nonlinearity, combined with the strong modal confinement due the large index contrast, yields nonlinear parameters that are typically 2 orders of magnitude larger than tapered silica fibers [7].

Previous experiments with chalcogenide planar waveguides $[8,9]$ have demonstrated the ability to engineer dispersion by controlling the waveguide geometry. Similarly, in a fiber waveguide, tapering provides the means to not only dramatically increase the nonlinear parameter of the fiber device $\gamma$, but to also engineer the total dispersion from normal to zero or even anomalous (see Fig. 1). This fact is crucial to using chalcogenide devices for supercontinuum generation in the telecommunications window because the material dispersion of chal cogenide at this wavelength is large and normal $(-360 \mathrm{ps} / \mathrm{nm} \mathrm{km})$. The tapering process used in this work end result is a developed $[10,11]$ for $\mathrm{As}_{2} \mathrm{Se}_{3}$ fiber. The of just $0.8 \mu \mathrm{m}^{2}$, which inegion with a mode area $\left(A_{\text {eff }}\right)$ $1550 \mathrm{~nm}$. Thu which increases $\gamma$ to $12,400 \mathrm{~W}^{-1} \mathrm{~km}^{-1}$ at petes well with the nonlinearity of the tapered fiber comSilica glass fiber motic construction techniques [12]. pulses into the leads were used to butt-couple the input the broad spechalcogenide fiber, and used to outcouple

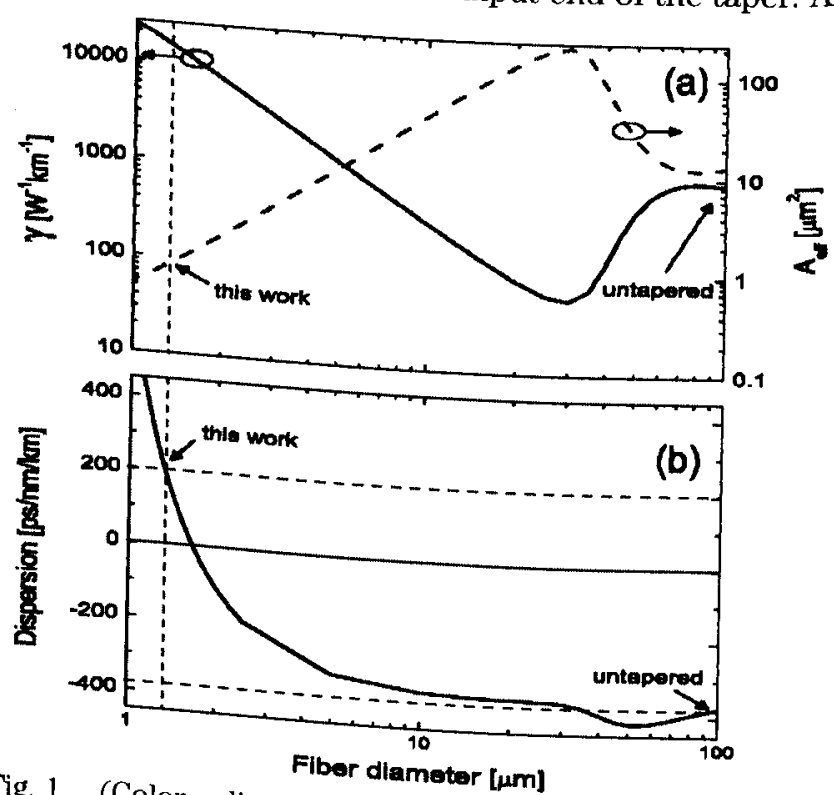

tical axis) and offine) Taper details. (a) Nonlinearity (left vertion of fiber diameter. effective mode area, and The nonlinear parameter mirrors the when the fiber diameter and reaches extremely large values only of the $\mathrm{As}_{2} \mathrm{~S}_{3}$ fiber as a function below $4 \mu \mathrm{m}$. (b) Dispersion profile fiber exhibits large, normal disper diameter. The untapered to introduce anomaloul dispersion. The effect of tapering is tually shifts the total dispersion to dispersion, which even20 total dispersion to zero and beyond. 
FC/APC connector is used to couple the laser output into the taper device, and the final result is a robust device that has $5.7 \mathrm{~dB}$ pigtail-to-pigtail insertion loss (split between $2.2 \mathrm{~dB}$ Fresnel loss and $3.5 \mathrm{~dB}$ propagation loss). In principle, the lumped propagation loss can be reduced through improvements in the tapering and silica-chalcogenide butt-coupling.

The input pulses to the $\mathrm{As}_{3} \mathrm{~S}_{3}$ fiber taper are generated from an erbium-fiber-based mode-locked laser (see Fig. 2). This laser is mode locked using the nonlinear polarization rotation scheme and produces 250 fs pulses at a $38.6 \mathrm{MHz}$ repetition frequency with $17 \mathrm{~mW}$ of average power. As the untapered region of the chalcogenide fiber presents significant normal dispersion, the input pulses to the taper device must be compensated by an equivalent amount of anomalous prechirp. The $\mathrm{As}_{2} \mathrm{~S}_{3}$ input fiber used in this experiment constitutes a net group-delay dispersion (GDD) of $0.114 \mathrm{ps}^{2}$. Experimentally, the best dispersion compensation (as judged by bandwidth produced) for this GDD required $5 \mathrm{~m}$ of SMF-28 fiber, in good agreement with the calculated ratio of dispersion and length (i.e. $\left|\beta_{2, \mathrm{As}, \mathrm{S}_{3}} / \beta_{2, \mathrm{SMF}-28}\right|=21.1$ and $l_{\mathrm{SMF}-28} / l_{\mathrm{As}_{2} \mathrm{~S}_{3}}$ $=20.2$ ). Frequency-resolved-optical-gating (FROG) measurements were performed on the chirped input pulses using a commercial FROG device. The recorded spectrograms and the corresponding time domain electric field envelopes of the direct laser output and the prechirped pulses can be seen in Fig. 3. These electric field envelopes were used as input pulses in the numerical modeling. The average power of the input pulses was tuned using an in-line variable optical attenuator.

Detection of the output spectra from the taper was accomplished using three separate spectrometers. The short wavelength side $(800-1200 \mathrm{~nm})$ was measured using an ANDO AQ6375, the middle wavelengths

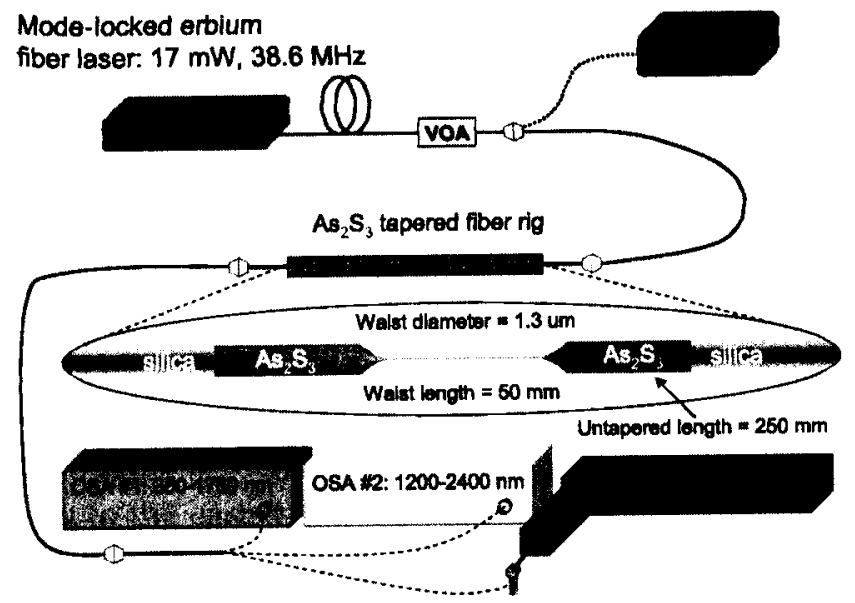

Fig. 2. (Color online) Experimental layout. Pulses from the erbium laser are prechirped using $5 \mathrm{~m}$ of silica fiber $\left(\beta_{2}=\right.$ $-0.021 \mathrm{ps}^{2} / \mathrm{m}$ ), while the variable optical attenuator (VOA) is used to vary the average power. A FROG is used to retrieve the full electric field that is used in the simulation. Light is coupled into the chalcogenide fiber via a butt-coupled silica fiber that is affixed with UV-cured epoxy. The output of the taper device is measured individually with three spectrometers covering various wavelength ranges. While optical spectrum analyzer (OSA) 1 and OSA 2 are fiber coupled, OSA 3 is free-space coupled and allows for sensitive detection of the long wavelength edge.

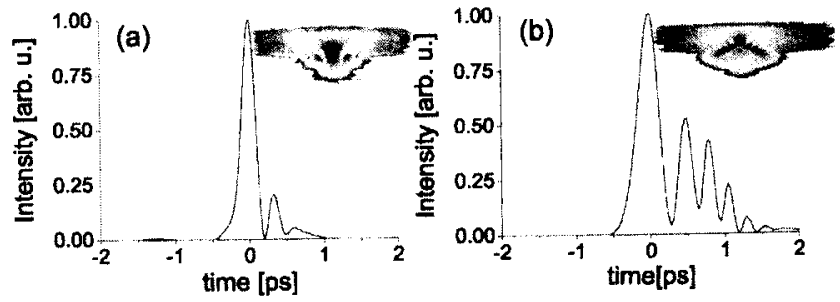

Fig. 3. (Color online) Experimental spectrograms and retrieved electric fields. (a) Shortest pulse achievable from the mode-locked laser. (b) Pulse as it appears just before entering the taper device. The pulse in (b) was used in the numerical model.

(1200-1900 nm) using a Yokogawa AQ6315, and the long wavelengths $(>1900 \mathrm{~nm})$ using a free-space scanning monochromator and a liquid-nitrogen-cooled mercurycadmium-telluride detector. While the measurement bandwidth of the Yokogawa was large enough to cover the range of interest (out to $2400 \mathrm{~nm}$ ), care had to be taken to avoid recording a false signal at the longer wavelengths due to the second-order diffraction from the grating. To avoid this, a $1500 \mathrm{~nm}$ long-pass filter was used in conjunction with the free-space spectrometer for the long wavelength regions.

The output spectra of the taper are shown in Fig. 4. As the power is incrementally increased, the spectrum
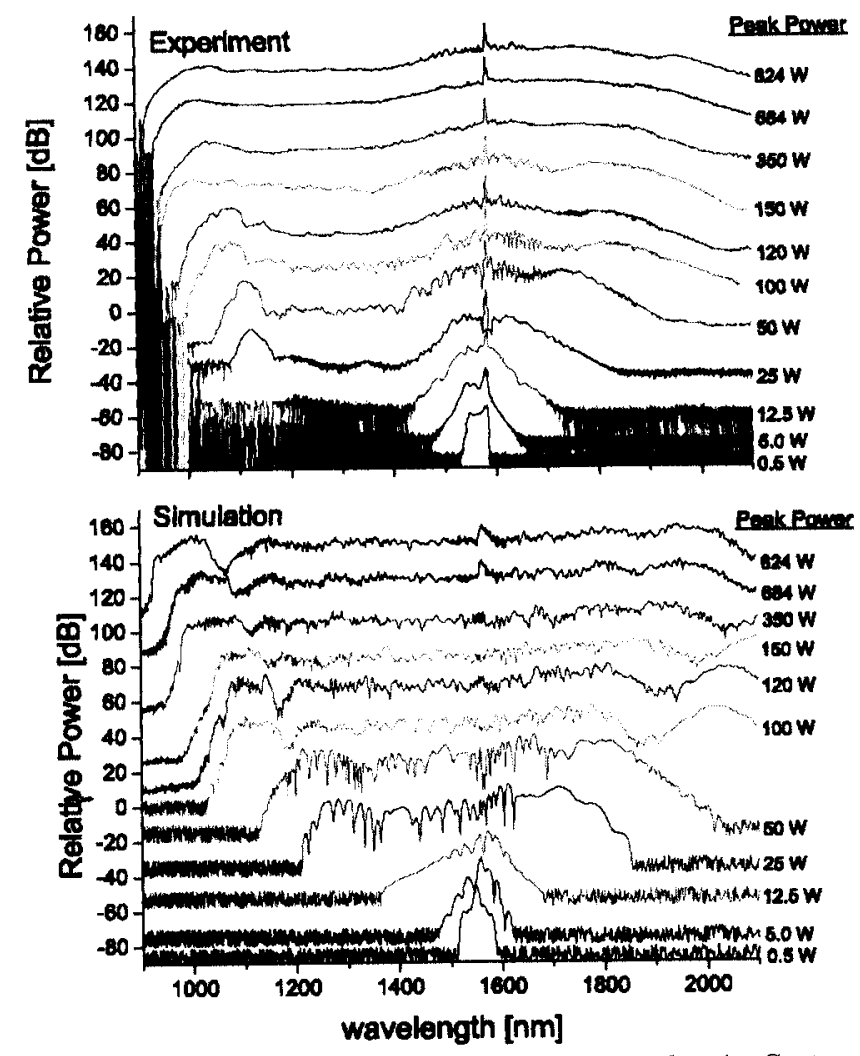

Fig. 4. (Color online) Output spectra from the $\mathrm{As}_{2} \mathrm{~S}_{3}$ taper (top) and numerical simulation (bottom). As the peak power is steadily increased, the supercontinuum generation achieves one octave of bandwidth $(-20 \mathrm{~dB}$ points $)$ at $150 \mathrm{~W}$ peak power. At the highest peak power $(824 \mathrm{~W})$, an octave is contained within the $-10 \mathrm{~dB}$ points, and the long wavelength edge is extending into the mid-IR regime $(>2 \mu \mathrm{m})$. The spike at the center of the spectra is a long pulse pedestal $(>10 \mathrm{ps}$ ) on the laser output, and has a minimal contribution to the broadening. 
begins to broaden to several hundred nanometers with relatively low peak powers, as has been previously reported [13]. With $77 \mathrm{pJ}$ of pulse energy (150 W peak power, $3 \mathrm{~mW}$ average power), the $-20 \mathrm{~dB}$ bandwidth covers the span from 970 to $1990 \mathrm{~nm}$, over one octave of optical bandwidth. The short wavelength, phase-matched dispersive wave is clearly visible at peak powers above $25 \mathrm{~W}$, while Raman shifting solitons are responsible for extending the bandwidth in the long wavelength side. For the highest peak power, the soliton number $(N=$ $\sqrt{L_{D} / L_{\mathrm{NL}}}$, where $L_{D}$ is the dispersion length and $L_{\mathrm{NL}}$ is the nonlinear length) is of the order of 50 , while the fission length is of the order of $5 \mathrm{~mm}$. This results in a large number of spectrally broad, overlapping soliton features [14] that shed energy into resonant dispersive waves over a wider bandwidth [15], while noise-induced fission of the initial pulse induces shot-to-shot variation, resulting in a smoothing effect that produces a remarkably flat spectrum (an octave is contained within the $-10 \mathrm{~dB}$ points). Using the measured taper parameters in conjunction with an adaptive split-step Fourier routine, we were able to accurately model the experimental results (Fig. 4, bottom) by numerically solving the nonlinear Schrödinger equation.

In conclusion, we have demonstrated an octave spanning spectrum in a sulfide-based chalcogenide tapered fiber using only $77 \mathrm{pJ}$ pulse energy, to our knowledge the lowest ever reported for octave generation in a taper [7]. These results were obtained by carefully tailoring the input pulse chirp and utilizing the robust power handling capabilities of sulfide. These results could find use in applications such as $f-2 f$ interferometers used in frequency combs. The taper coupling method presented here allows the experimenter to easily and efficiently couple light into the taper via standard APC connectors. In addition, the low power required for octave generation in this taper relaxes the power requirement of standard octave spanning generation [16], and could potentially remove the need for an amplifier in such a system. Future work will involve investigating the long wavelength side of the spectral generation for mid-IR applications. Through dispersion engineering in the taper, it may be possible to utilize the soliton self-frequency shift to achieve highly efficient spectral shifting to wavelengths beyond $2 \mathrm{um}$.
Funding from the Australian Research Council (ARC) through its Federation Fellow and Centres of Excellence programs is gratefully acknowledged. The Centre for (CUDOS) is dwidth Devices for Optical Systems (CUDOS) is an ARC Centre of Excellence.

\section{References}

1. I. Hartl, X. D. Li, C. Chudoba, R. K. Ghanta, T. H. Ko, J. G. Fujimoto, J. K. Ranka, and R. S. Windeler, Opt. Lett. 26,

M. J. Thorpe, D. D. Hudson, K. D. Moll, J. Lasri, and J. Ye, S. T. Lett. 32, 307 (2007)

4. D. J. Jones, S. J. Ye, Rev. Mod. Phys. 75, 325 (2003). Windeler, J. L. A. Diddams, J. K. Ranka, A. Stentz, R. S. (2000).
(J. L. Hall, and S. T. Cundiff, Science 288, 635

(2007).
N. Newbury and W. Swann, J. Opt. Soc. Am. B 24, 1756

6. Focus (2011).

7. M. A (2004). 8. M. R. E. Lamont, B. Luther-Davies, D.-Y. Choi, S. (2008).

9. N.

Osellame, R. Thomson, H. Bookey, S. Shen, N. Chiodo, R. 15, 15776 (2007). 10. D. Yeom

Fu, and B. J. Mägi, M. R. E. Lamont, M. A. F. Roelens, L.

11. E. C. Mägi, L. Eggleton, Opt. Lett. 33, 660 (2008). and B. J. Eggleton, H. C. Nguyen, M. R. Lamont, D. I. Yeom,

12. M. Liao, G. Egleton, Opt. Express 15, 10324 (2007). Lightwave Techn, X. Yan, T. Suzuki, and Y. Ohishi, J. 13. S. Dekker C. Xiong. 29, 194 (2011).

B. Shaw, I. D. Xiong, E. Mägi, A. C. Judge, J. S. Sanghera, L. Proceedings of Conferenal, D. J. Moss, and B. J. Eggleton, in Quantum Electronicence on Lasers and Electro-Optics/ 2010 (Optical Sonics and Laser Science Conference

14. J. Herrmann, U. Griety of America, 2010), paper CMM6. Nickel, J. C. Knight W. J. Zhavoronkov, A. Husakou, D. G. Korn, Phys. Rev W. J. Wadsworth, P. St. J. Russell, and 5. J. Dudley, G. Genty, Lett. 88, 173901 (2002). (2006).

16. J. W. DiMarcello, E. Monberg, A. Yablon, C. Jorgensen, and T.
Veng, Opt Veng, Opt. Lett. 28, 643 (2003). 